Commentary on Koonin's paper about CRISPR-Cas

Biology \& Philosophy

\title{
CRISPR-Cas immunity: Beyond nonself and defence
}

Thomas Pradeu ${ }^{1,2}$ \& Jean-François Moreau ${ }^{1,3}$

1. ImmunoConcept, UMR5164, CNRS \& University of Bordeaux, France

2. Institut d'histoire et de philosophie des sciences et des techniques (IHPST), UMR8590,

CNRS \& Panthéon-Sorbonne University, Paris, France

3. Department of Immunology, CHU Bordeaux Hospital, Bordeaux, France

\section{Keywords}

CRISPR-Cas; immune system; self; nonself; phage; virus; defence; repair; autoimmunity; tolerance; Bacteria; Archaea, microbiota.

\section{Abstract}

In this commentary of Koonin's target paper, we defend an extended view of CRISPR-Cas immunity by arguing that CRISPR-Cas includes, but cannot be reduced to, defence against nonself. CRISPR-Cas systems can target endogenous elements (for example in DNA repair) and tolerate exogenous elements (for example some phages). We conclude that the vocabulary of "defence" and "nonself" might be misleading when describing CRISPR-Cas systems. 


\section{Introduction}

Eugene Koonin has put forward very original and stimulating ideas about CRISPR-Cas systems in his target paper for Biology \& Philosophy (Koonin 2018, this issue) as well as in previous publications (Makarova et al. 2011; Koonin 2017; Koonin et al. 2017). In this commentary, we argue that CRISPR-Cas includes, but cannot be reduced to, defence against nonself. This claim is not intended as an objection to Koonin, but rather as an invitation to fully clarify to what extent the "expanded" view of CRISPR-Cas immunity suggested here is adequate.

This paper does not address some of the central claims made by Koonin on CRISPR-Cas systems in his paper, including the possibility to describe this process as "Lamarckian". These points are examined by other commentaries of this journal issue. Rather, as an immunologist and a philosopher of immunology, our focus is on the description of CRISPR-Cas as an immune system, and on the question of how to best characterize this immune system. We try to make use of our knowledge of the functioning of other immune systems in the living world to better identify the characteristics that are specific to CRISPR-Cas, and those that it shares with other immune systems.

CRISPR-Cas constitutes a fantastic discovery for the field of immunology, in that it brilliantly confirms that immune systems, far from being limited to vertebrates, are found everywhere across the living world - in animals, plants, Bacteria and Archaea (Shabalina and Koonin 2008; Pradeu 2012; Koonin and Krupovic 2015). The description of CRISPR-Cas as an immune system was not obvious fifteen years ago, and this idea has a convoluted history (Morange 2015; Ledford 2016; Barrangou and Horvath 2017; Ishino et al. 2018). Although several groups had anticipated it, this idea was stated clearly in three papers published only in 2005 (Mojica et al. 2005; Pourcel et al. 2005; Bolotin et al. 2005) and 2006 (Makarova et al. 2006), and it is often considered that it has been experimentally demonstrated only in 2007 by the group of Philippe Horvath in Science, where CRISPR-Cas was proved to provide acquired and specific resistance to phages in Bacteria and Archaea (Barrangou et al. 2007).

The long-dominant view in immunology has been that an immune system is a defence system that preserves its host (the "self") while eliminating every foreign entity ("nonself") (Burnet 1960; Bretscher and Cohn 1970; Clark 2008). Unsurprisingly, the characterization of CRISPRCas as a prokaryote immune system has been done using the vocabulary of "defence" and "self" vs. "foreign" (e.g., (Barrangou et al. 2007; Levy et al. 2015)), and this vocabulary is also prevalent in Koonin's target paper (Koonin 2018, this issue).

The last twenty years of research, though, have cast doubt on the classic view of immune systems as defence systems against nonself (Pradeu 2012), by showing that:

i) the immune system plays a key role in the construction (development) and maintenance (repair and "housekeeping" mechanisms) of the organism, and therefore is not limited to defence activities.

ii) many endogenous ("self") components trigger an effector immune response.

iii) many exogenous ("nonself") components do not trigger an effector immune response and are actively tolerated by the host.

These observations have been confirmed across multicellular species (including vertebrates, invertebrates, and plants). They have led to the formulation of new conceptual and theoretical frameworks, distinct from the classic self-nonself view (Matzinger 1994; Cohen 2000; Pradeu and Carosella 2006). Hence, it seems worth asking whether CRISPR-Cas can be described using a framework that would differ from the traditional concept of defence of the self against 
nonself. We therefore raise in what follows two intimately related questions: does CRIPSR-Cas respond to nonself?; is CRISPR-Cas a system of defence?

\section{Does CRISPR-Cas respond to nonself?}

Koonin describes CRISPR-Cas as a system that defends its host against nonself, while preserving the self. The idea seems rather intuitive: the CRISPR-Cas system would have evolved to recognize genetically foreign elements, enabling the concerned Bacteria and Archaea to eliminate phages and other mobile elements while avoiding any attack against its own genetic material. Given the intuitive character of this concept and the long tradition of interpreting immune response in terms of self-nonself since at least Burnet, it is not surprising that this description is dominant in the literature on CRISPR-Cas-based immunity (Barrangou et al. 2007; Marraffini and Sontheimer 2010; Levy et al. 2015; Nuñez et al. 2015).

Yet, in other organisms, the long-dominant and also very intuitive idea that immune responses target the nonself has been strongly criticized in the last thirty years or so (Tauber 1994; Matzinger 1994; Cohen 2000; Pradeu and Carosella 2006). First, many endogenous (self) components trigger an effector immune response, giving rise to non-pathogenic autoimmunity. This normal autoimmunity is distinct from the pathological case of autoimmune diseases. Normal autoimmunity is in fact indispensable for the normal functioning of the organism. For example, phagocytes such as macrophages ensure the disposal of dead cells, a phenomenon that occurs at a large scale and is pivotal for tissue maintenance (Savill and Fadok 2000) as well as autoimmunity avoidance (Gordon and Plüddemann 2018). More generally, entire circuits and responses of the immune system are based on self recognition (Hayday 2009; Yu et al. 2015). Second, many exogenous (nonself) components do not trigger an effector immune response and are actively tolerated by the host, such as many components of the microbiota (Hooper and Gordon 2001; Donaldson et al. 2018).

Could the same be true for CRISPR-Cas? In other words, could the CRISPR-Cas system sometimes target "self" elements, and preserve "nonself" elements? We believe that this is indeed likely to be so. First, forms of autoimmunity in CRISPR-Cas and in other immune-like mechanisms in prokaryotes have been described. For example, (Stern et al. 2010) propose that leaky incorporation of self nucleic acids into CRISPR arrays could explain the self-targeting of CRISPR found in 59 of 330 (18\%) of the CRISPR-encoding organisms. Obviously, this could lead to a fitness cost for the organism, but this is not what is observed, as in most cases a degradation of CRISPR activity occurs. Second, forms of "tolerance to nonself" in CRISPR-Cas systems have been documented. Marraffini's group has recently shown that type III-A CRISPR immunity in Staphylococcus epidermidis prevents lytic infection while tolerating lysogenization by temperate phages (Goldberg et al. 2014). Conditional tolerance is mediated by transcription-dependent DNA targeting and ensures that targeting is resumed when the prophage lytic cycle is induced. The same group proposes that CRISPR-Cas systems have been optimized for selective incorporation of foreign genetic information, enabling a balance between resistance to pathogens and tolerance to favourable exogenous elements (Goldberg and Marraffini 2015).

We believe that further evidence of physiological autoimmunity and tolerance to nonself in CRISPR-Cas systems will probably accumulate in the near future. If true, it will confirm what has now been demonstrated in a multitude of other immune systems, namely that the 
targeting of the self and the preservation of the nonself are normal, even indispensable, phenomena ${ }^{1}$.

\section{Is CRISPR-Cas a system of defence?}

Our second question is intimately related to the first. One crucial set of circumstances in which immune responses to the self have been documented is tissue repair (Wynn et al. 2013; Eming et al. 2017). In addition to the damages inflicted by the invader, a system that "fights" external threats also causes important damages, hence the need to evolve repair mechanisms, enabling to maintain the integrity and cohesion of the organism (e.g., (Bouchery and Harris 2017)). More generally, it is increasingly recognized that immune systems are involved in many physiological activities beyond defence. This includes development and maintenance activities, such as repair and "housekeeping" mechanisms.

Could this be also true of CRISPR-Cas? The answer seems at least in part to be positive (Hille et al. 2018). In addition to defence, recent evidence shows that CRISPR-Cas systems modulate other processes, such as the genetic regulation of group behaviour and virulence, DNA repair, and genome evolution (Westra et al. 2014). For example, Cas1 of the E. coli type I-E system interacts with DNA-recombination and DNA-repair enzymes, and deleting cas1 or the associated CRISPR loci leads to increased sensitivity to DNA-damaging agents as well as dysfunctional chromosome segregation (Babu et al. 2010).

Remarkably, Koonin's group was among the first to suggest that CRISPR-Cas could be a repair system (Makarova et al. 2002). Koonin was later involved in the description of CRISPR-Cas as a dual system - participating in both defence and repair (Babu et al. 2010), which is exactly the idea we think adequate, even though Koonin (2018, this issue) suggests that the insistence on the repair activities of CRISPR-Cas "missed the mark". The very simple question we raise here is whether describing CRISPR-Cas systems as systems of defence against nonself tends to keep hidden its other, equally important, activities.

\section{Conclusion}

A hundred years of research in immunology oriented towards the idea that immunity is the defence of the self against the nonself has given rise to a mindset: when novel immune systems are identified, scientists tend to describe them using this pre-existing conceptual framework. Nevertheless, in the same way as the immune systems of animals and plants cannot be solely understood as a form of defence against nonself, it seems to us that this concept does not give justice to the complexities of the CRISPR-Cas systems in Archaea and Bacteria either. The activities of CRISPR-Cas systems go well beyond mere defence, and they do not necessarily target nonself. We suggest that CRISPR-Cas actually detects genetic anomalies, regardless of their origin ("self" or "nonself"). This approach might not only help draw important parallels between CRISPR-Cas and other immune systems and eventually build a unified account of immunity across all forms of life, but also open new avenues for experimental and conceptual investigations.

\footnotetext{
${ }^{1}$ In some cases, including CRISPR-Cas systems, tolerated foreign elements can even be transmitted to the offspring, allowing adaptations at the level of populations and/or species. 


\section{Acknowledgments}

We would like to thank Michael Weisberg for the opportunity to write about the philosophical and immunological dimensions of the CRISPR-Cas system, and Eugene Koonin for his extremely innovative and stimulating ideas.

T. P. has received funding from the European Research Council (ERC) under the European Union's Horizon 2020 research and innovation programme - grant agreement $n^{\circ} 637647$ IDEM.

\section{References}

Babu M, Beloglazova N, Flick R, et al (2010) A dual function of the CRISPR-Cas system in bacterial antivirus immunity and DNA repair. Molecular Microbiology 79:484-502. doi: 10.1111/j.1365-2958.2010.07465.x

Barrangou R, Fremaux C, Deveau H, et al (2007) CRISPR Provides Acquired Resistance Against Viruses in Prokaryotes. Science 315:1709-1712. doi: 10.1126/science.1138140

Barrangou R, Horvath P (2017) A decade of discovery: CRISPR functions and applications. Nature Microbiology 2:nmicrobiol201792. doi: 10.1038/nmicrobiol.2017.92

Bolotin A, Quinquis B, Sorokin A, Ehrlich SD (2005) Clustered regularly interspaced short palindrome repeats (CRISPRs) have spacers of extrachromosomal origin. Microbiology (Reading, Engl) 151:2551-2561. doi: 10.1099/mic.0.28048-0

Bouchery T, Harris NL (2017) Specific repair by discerning macrophages. Science 356:10141014. doi: 10.1126/science.aan6782

Bretscher P, Cohn M (1970) A theory of self-nonself discrimination. Science 169:1042-1049

Burnet FM (1960) Immunological recognition of self. Nobel Lectures in Physiology or Medicine 3:689-701

Clark WR (2008) In Defense of Self: How the Immune System Really Works. Oxford University Press, New York

Cohen IR (2000) Discrimination and dialogue in the immune system. Semin Immunol 12:215219; discussion 257-344. doi: 10.1006/smim.2000.0234

Donaldson GP, Ladinsky MS, Yu KB, et al (2018) Gut microbiota utilize immunoglobulin A for mucosal colonization. Science 360:795-800. doi: 10.1126/science.aaq0926

Eming SA, Wynn TA, Martin P (2017) Inflammation and metabolism in tissue repair and regeneration. Science 356:1026-1030. doi: 10.1126/science.aam7928

Goldberg GW, Jiang W, Bikard D, Marraffini LA (2014) Conditional tolerance of temperate phages via transcription-dependent CRISPR-Cas targeting. Nature 514:633-637. doi: 10.1038/nature13637

Goldberg GW, Marraffini LA (2015) Resistance and tolerance to foreign elements by prokaryotic immune systems - curating the genome. Nature Reviews Immunology 15:717724. doi: $10.1038 /$ nri3910

Gordon S, Plüddemann A (2018) Macrophage Clearance of Apoptotic Cells: A Critical Assessment. Front Immunol 9:. doi: 10.3389/fimmu.2018.00127

Hayday AC (2009) Gammadelta T cells and the lymphoid stress-surveillance response. Immunity 31:184-196. doi: 10.1016/j.immuni.2009.08.006

Hille F, Richter H, Wong SP, et al (2018) The Biology of CRISPR-Cas: Backward and Forward. Cell 172:1239-1259. doi: 10.1016/j.cell.2017.11.032

Hooper LV, Gordon JI (2001) Commensal host-bacterial relationships in the gut. Science 


\section{2:1115-1118}

Ishino Y, Krupovic M, Forterre P (2018) History of CRISPR-Cas from Encounter with a Mysterious Repeated Sequence to Genome Editing Technology. J Bacteriol 200:. doi: 10.1128/JB.00580-17

Koonin EV (2017) Evolution of RNA- and DNA-guided antivirus defense systems in prokaryotes and eukaryotes: common ancestry vs convergence. Biology Direct 12:5. doi: 10.1186/s13062-017-0177-2

Koonin EV, Krupovic M (2015) Evolution of adaptive immunity from transposable elements combined with innate immune systems. Nat Rev Genet 16:184-192. doi: 10.1038/nrg3859 Koonin EV, Makarova KS, Zhang F (2017) Diversity, classification and evolution of CRISPR-Cas systems. Curr Opin Microbiol 37:67-78. doi: 10.1016/j.mib.2017.05.008 Ledford H (2016) The unsung heroes of CRISPR. Nature 535:342-344. doi: 10.1038/535342a Levy A, Goren MG, Yosef I, et al (2015) CRISPR adaptation biases explain preference for acquisition of foreign DNA. Nature 520:505-510. doi: 10.1038/nature14302

Makarova KS, Aravind L, Grishin NV, et al (2002) A DNA repair system specific for thermophilic Archaea and bacteria predicted by genomic context analysis. Nucleic Acids Res 30:482-496

Makarova KS, Grishin NV, Shabalina SA, et al (2006) A putative RNA-interference-based immune system in prokaryotes: computational analysis of the predicted enzymatic machinery, functional analogies with eukaryotic RNAi, and hypothetical mechanisms of action. Biol Direct 1:7. doi: 10.1186/1745-6150-1-7

Makarova KS, Haft DH, Barrangou R, et al (2011) Evolution and classification of the CRISPRCas systems. Nat Rev Microbiol 9:467-477. doi: 10.1038/nrmicro2577

Marraffini LA, Sontheimer EJ (2010) Self versus non-self discrimination during CRISPR RNAdirected immunity. Nature 463:568-571. doi: 10.1038/nature08703

Matzinger P (1994) Tolerance, Danger, and the Extended Family. Annu Rev Immunol 12:9911045. doi: 10.1146/annurev.iy.12.040194.005015

Mojica FJM, Díez-Villaseñor C, García-Martínez J, Soria E (2005) Intervening sequences of regularly spaced prokaryotic repeats derive from foreign genetic elements. J Mol Evol 60:174-182. doi: 10.1007/s00239-004-0046-3

Morange M (2015) What history tells us XXXVII. CRISPR-Cas: The discovery of an immune system in prokaryotes. J Biosci 40:221-223

Nuñez JK, Harrington LB, Kranzusch PJ, et al (2015) Foreign DNA capture during CRISPR-Cas adaptive immunity. Nature 527:535-538. doi: 10.1038/nature15760

Pourcel C, Salvignol G, Vergnaud G (2005) CRISPR elements in Yersinia pestis acquire new repeats by preferential uptake of bacteriophage DNA, and provide additional tools for evolutionary studies. Microbiology (Reading, Engl) 151:653-663. doi: 10.1099/mic.0.27437-0 Pradeu T (2012) The Limits of the Self: Immunology and Biological Identity. Oxford University Press, New York

Pradeu T, Carosella E (2006) On the definition of a criterion of immunogenicity. Proceedings of the National Academy of Sciences USA 103:17858-17861

Savill J, Fadok V (2000) Corpse clearance defines the meaning of cell death. Nature 407:784788. doi: $10.1038 / 35037722$

Shabalina SA, Koonin EV (2008) Origins and evolution of eukaryotic RNA interference. Trends in Ecology \& Evolution 23:578-587. doi: 10.1016/j.tree.2008.06.005

Stern A, Keren L, Wurtzel O, et al (2010) Self-targeting by CRISPR: gene regulation or autoimmunity? Trends in Genetics 26:335-340. doi: 10.1016/j.tig.2010.05.008 
Tauber Al (1994) The Immune Self: Theory or Metaphor? Cambridge University Press, Cambridge

Westra ER, Buckling A, Fineran PC (2014) CRISPR-Cas systems: beyond adaptive immunity. Nat Rev Micro 12:317-326. doi: 10.1038/nrmicro3241

Wynn TA, Chawla A, Pollard JW (2013) Macrophage biology in development, homeostasis and disease. Nature 496:445-455. doi: 10.1038/nature12034

Yu W, Jiang N, Ebert PJR, et al (2015) Clonal Deletion Prunes but Does Not Eliminate SelfSpecific $\alpha \beta$ CD8(+) T Lymphocytes. Immunity 42:929-941. doi:

10.1016/j.immuni.2015.05.001 